\title{
Wideband Differentially-Fed Slot Antenna and Array With Circularly Polarized Radiation for Millimeter-Wave Applications
}

\author{
Lehu Wen, Steven Gao, Fellow, IEEE, Wei Hu, Member, IEEE, Qi Luo, Senior Member, IEEE, \\ Xue-Xia Yang, Senior Member, IEEE, and Benito Sanz-Izquierdo
}

\begin{abstract}
A wideband differentially-fed slot antenna is presented for millimeter-wave (mmWave) applications. A novel method of using stepped corner-shaped slot is first utilized to establish the wideband circularly polarized $(\mathrm{CP})$ radiation. In the configuration of corner-shaped slot, two wide open slots at the ends are utilized for effective orthogonal radiation, while the narrow slot at the center is utilized for power transmission and quadrature phase delay. An equivalent circuit is given to illustrate the inner working principle for $\mathrm{CP}$ radiation. In addition, square cuts are etched on the four corners of the radiating patches to further increase the axial ratio (AR) and impedance bandwidth. Based on this design concept, the antenna element was first designed and fabricated for performance verification. Then, a $1 \times 4$ linear array with beam scanning performance and a $4 \times 4$ planar array with high gain and stable radiation were designed and fabricated. Both the simulated and measured results show that the $1 \times 4$ linear array and $4 \times 4$ planar array can have wide overlapped impedance and AR bandwidths of $30.6 \%$ and $33.6 \%$ with thickness of $0.16 \lambda_{0}$. The advantages of compact size and wide bandwidth make the presented antenna a good candidate for mmWave applications.
\end{abstract}

Index Terms - Circular polarization, compact antenna, slot antenna, wideband antenna.

\section{INTRODUCTION}

$\mathrm{W}$ ITH the fast development of wireless communication systems, millimeter-wave (mmWave) technology plays an important role in these systems owing to its capabilities of providing wide available communication bandwidth, high data rate, and large system capacity. Therefore, many wireless communication systems are designed in mmWave frequency band, such as satellite communications, autonomous vehicle radars, 5G mobile communications [1], etc. Because of the natural immunity of polarization direction and advantages of the reduced multi-path fading effect and increased channel

This work was supported in part by EPSRC grants EP/N032497/1, in part by EP/P015840/1, and in part by EP/S005625/1. (Corresponding author: Lehu Wen.)

L. Wen, S. Gao, and B. Sanz-Izquierdo are with the School of Engineering and Digital Arts, University of Kent, Canterbury, CT2 7NT, U.K. (e-mail: L.Wen@kent.ac.uk)

W. Hu is with the National Key Laboratory of Antennas and Microwave Technology, Xidian University, Xian, 710071, China.

Q. Luo is with the School of Physics, Engineering and Computer Science, University of Hertfordshire, Hatfield, AL10 9AB, UK.

X.-X. Yang is with the School of Communication and Information Engineering, Shanghai University, 200444, China. capacity, circularly polarized (CP) antennas are widely used in these wireless communication systems [2].

A common way to realize wideband $\mathrm{CP}$ radiation is utilizing crossed dipoles [3]-[10]. By integrating a narrow loop-shaped phase delay line, parasitic patches, or shorted plates for bandwidth enhancement, wide axial ratio (AR) bandwidth of up to $106 \%$ is realized in [3]-[4]. Different types of feed networks can also be utilized for wideband CP radiation [5]-[8]. In detail, series-feed networks [5]-[6], Wilkinson power divider loaded with open-circuited and short-circuited stubs [7]-[8] are utilized into CP antenna designs. Specifically, a wideband CP antenna utilizing Schiffman phase shifters [8] achieves a very wide AR bandwidth of $96 \%$. In addition, meta-material unit cells [9]-[10] can be loaded as phase delay lines for wideband $\mathrm{CP}$ radiation. The developed antenna in [10] utilizes left-hand and right-hand transmission line unit cells for wideband quadrature excitation, thus wide AR bandwidth of less than $1.7 \mathrm{~dB}$ is achieved. These above antennas normally can have a wide bandwidth for $\mathrm{CP}$ radiation. However, due to the large profiles and feeding methods of using coaxial cables or vertical baluns, it is difficult to directly apply these designs in mmWave applications.

To realize CP radiation for mmWave applications, a convenient way is to excite the top patch using a microstrip line [11]-[12]. In addition, substrate integrated waveguide (SIW) can be used to drive the patch, slot, and aperture [13]-[16]. In [16], curved microstrip line is designed as radiator and SIW is used as the series feeding lines for single-layer traveling-wave $\mathrm{CP}$ radiation. However, travelling-wave $\mathrm{CP}$ antennas normally suffer narrow impedance and AR bandwidths. To improve the impedance and AR bandwidths, multi-layer substrates are used in the CP antenna design [17]-[19]. In [18], magneto-electric dipole excited by a slot aperture is utilized for wideband $\mathrm{CP}$ array design. However, as the increase of substrate layers, blind and buried via holes will be introduced in some of these designs, which complicates the stacked structure and increases the fabrication cost. Recently, owing to the advantages of high common mode suppression and low noise level, differential electric ICs are widely used in the microwave circuit designs. When traditional single-ended antennas are connected to these differential ICs, baluns or differential feed networks will be incorporated to convert the single-ended signals into differential signals. This will cause additional insertion loss and IC cost for signal conversion. Therefore, differentially-fed antennas [20]-[23] are developed to directly match these differential ICs. 
In this work, a wideband differentially-fed slot antenna and array are presented for mmWave applications. A novel design method of using corner-shaped stepped slot is utilized to realize wideband $\mathrm{CP}$ radiation. The stepped slot consists of two wide open slots and a center narrow slot. By elaborately changing the width and length of the narrow slot, equal magnitude and quadrature phase excitation is established for $\mathrm{CP}$ radiation. To further improve the AR and impedance bandwidth, four square cuts are etched on the corners of the radiating patches. Based on the presented low-profile $\mathrm{CP}$ antenna element, a $1 \times 4$ linear array with beam-scanning performance and a $4 \times 4$ planar array with high and stable antenna gain were designed, fabricated, and measured. Both the measured and simulated results show that wide overlapped $\mathrm{AR}$ and impedance bandwidths of 23.8-32.4 GHz (30.6\%) and 22.8-32 GHz (33.6\%) are achieved for the fabricated linear array and planar array. The designed scanning beams can cover $-27^{\circ}$ to $-27^{\circ}$ for the fabricated $1 \times 4$ linear array, and high and stable gain of 17.1-18.5 dBic is achieved for the fabricated $4 \times 4$ planar array.

This paper is organized as follows. Section II presents the design of the wideband CP antenna element. Section III illustrates the design of $1 \times 4$ linear array with beam scanning performance. Section IV elaborates the design of a high gain $4 \times 4$ planar array. Finally, conclusions are drawn in Section V.

\section{ELEMENT Design}

In this section, a novel method of using corner-shaped stepped slot is utilized for wideband $\mathrm{CP}$ radiation. This differentially-fed CP antenna was then designed, fabricated, and measured as a basis for the following array design.

\section{A. Antenna Configuration}

The configuration of the proposed differentially-fed CP antenna is shown in Fig. 1. Fig. 1 (a) shows the top view and side view of the presented antenna. It can be seen that the presented mmWave $\mathrm{CP}$ antenna is composed of four different conducting layers. Each layer is etched on the top and bottom of two substrates. Commercially available substrates of RO4003C from Rogers Corporation with the relative permittivity of 3.55, loss tangent of 0.0027 , and thicknesses of $0.508 \mathrm{~mm}$ and $0.305 \mathrm{~mm}$ are used in the antenna design. The copper thickness of each layer is $0.017 \mathrm{~mm}$, which is considered in the antenna simulation. A rigid foam of Rohacell 51-HF from Emkay plastics Ltd with the relative permittivity of 1.06 and thickness of $1 \mathrm{~mm}$ is inserted between the conducting layer 2 and layer 3 . In the design of the antenna, EM simulation software of ANSYS electromagnetic suite 18.0 is used for performance evaluation.

Fig. 1 (b) shows the detailed configuration in each conducting layer. The first layer has two stair-shaped feed lines, which are utilized to excite the bottom wide open-slots properly for $\mathrm{CP}$ radiation, and also to achieve wide impedance bandwidth. The second layer is the main radiator of the antenna. There are four pieces of copper sheets with one opposite-pair shorted at the center, while another opposite-pair left open at the center. This configuration builds up two symmetrical corner-shaped stepped slots. These slots have two important functions. First, four wide open-slots (open slots 1-4) with the width W2 and length L4 are excited for effective orthogonal radiation. Then, two narrow slot-typed transmission lines with the width W5 and length L5 are designed at the center for the quadrature phase shift. To further reduce the radiator size and increase the operation bandwidth, four square-shaped cuts with the length of W4 are etched on the four corners of the radiating patches. Conducting layer 3 is the ground plane etching with only isolating circles, which are used to avoid the electrical connection from the plated vias. Conducting layer 4 is printed with a pair of differential $50 \Omega$ microstrip feed lines. The input energy is transmitted from the bottom feed lines to the top feed lines through two conducting vias. The detailed parameters of the proposed antenna are listed in the caption of Fig. 1 for ease of reference.

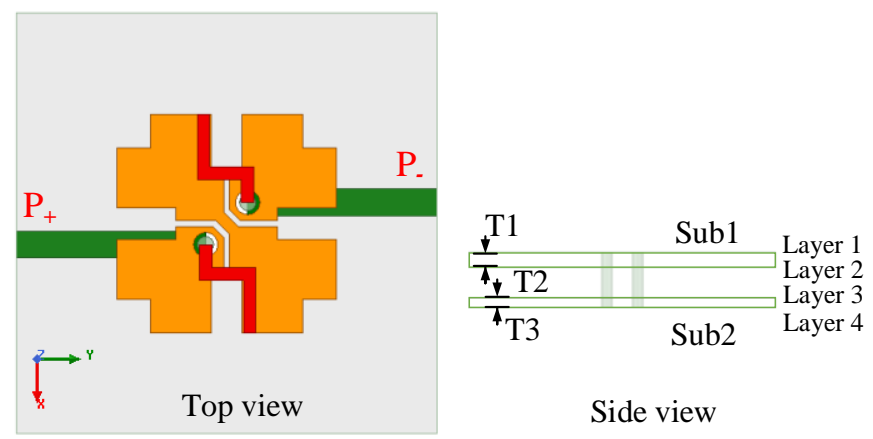

(a)

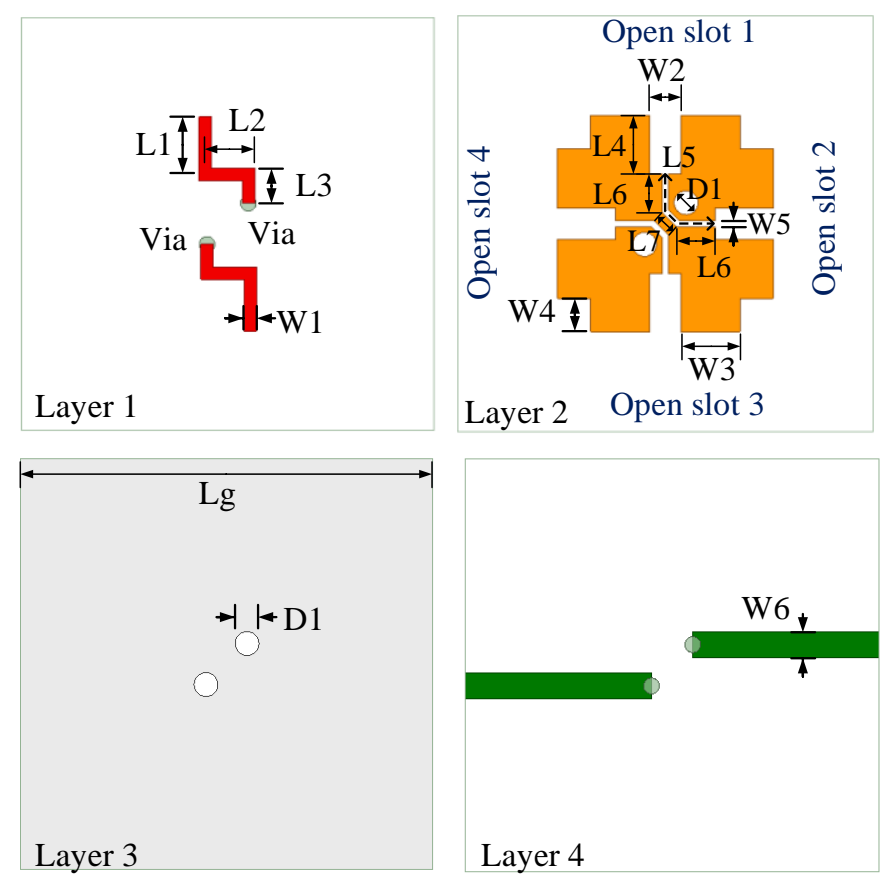

(b)

Fig. 1. Configuration of the proposed differentially-fed CP antenna. (a) Top view and side view. (b) Configuration in each layer. (Detailed parameters, $\mathrm{T} 1=0.508 \mathrm{~mm}, \mathrm{~T} 2=1 \mathrm{~mm}, \mathrm{~T} 3=0.305 \mathrm{~mm}, \mathrm{~W} 1=0.3 \mathrm{~mm}, \mathrm{~W} 2=0.75 \mathrm{~mm}, \mathrm{~W} 3=1.4$ $\mathrm{mm}, \mathrm{W} 4=0.8 \mathrm{~mm}, \mathrm{~W} 5=0.15 \mathrm{~mm}, \mathrm{~W} 6=0.64 \mathrm{~mm}, \mathrm{~L} 1=1.4 \mathrm{~mm}, \mathrm{~L} 2=1.1 \mathrm{~mm}$, L3 $=0.7 \mathrm{~mm}, \mathrm{~L} 4=1.4 \mathrm{~mm}, \mathrm{~L} 5=2.2 \mathrm{~mm}, \mathrm{~L} 6=0.9 \mathrm{~mm}, \mathrm{~L} 7=0.4 \mathrm{~mm}, \mathrm{D} 1=0.6 \mathrm{~mm}$, $\mathrm{Lg}=20 \mathrm{~mm}$.)

\section{B. Principle of CP Radiation}

In the design of the presented antenna, two different types of slots are first introduced for the ideal CP radiation. One is the 
wide open slots for orthogonal radiation, the other is the narrow slotline working for the power transmission and quadrature phase shift. To illustrate the $\mathrm{CP}$ working principle of this antenna, its equivalent circuit is shown in Fig. 2. In the equivalent circuit, the parallel lumped components of $L_{s}, C_{s}$, and $R_{S}$ represent the resonances caused by the wide open slots. The input impedance of the wide open slot is denoted as $Z_{s}$. The characteristic impedance and phase of the narrow slotline are denoted as $Z_{1}$ and $\theta_{1} . Z_{0}$ is the characteristic impedance of the top microstrip feed line. Based on this equivalent circuit, the transmission coefficients of the three-port network at nodes 1,2 , and 3 can be obtained as below (detailed derivation of the following equations is illustrated in Appendix).

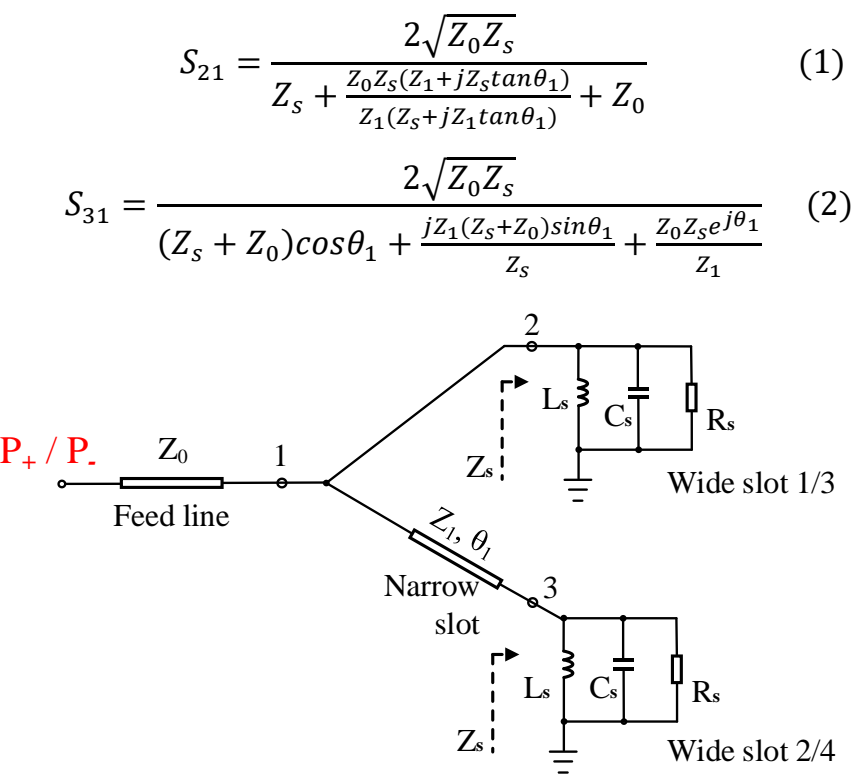

Fig. 2. Equivalent circuit of the presented CP antenna.

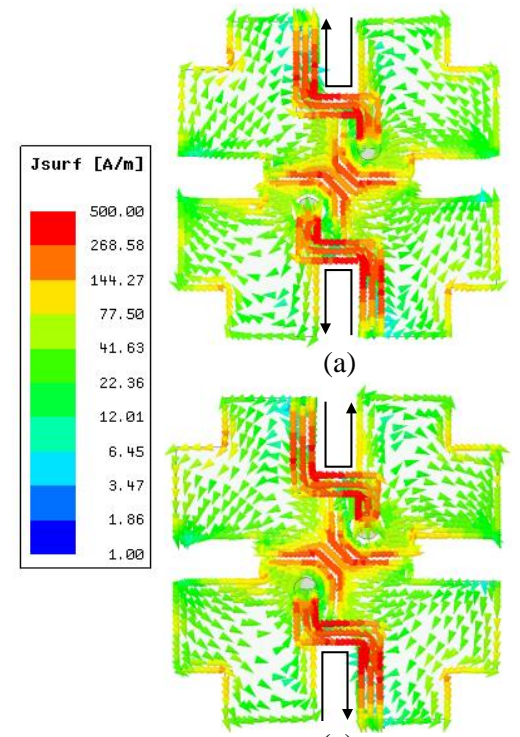

(c)

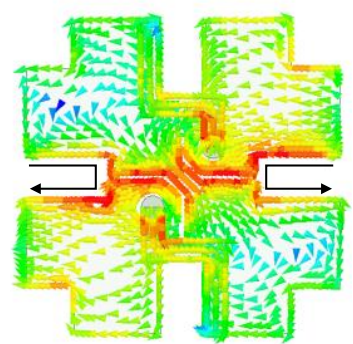

(b)

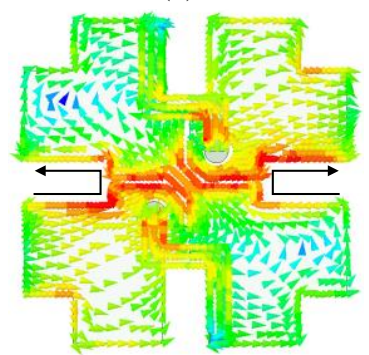

(d)
Fig. 3. Current distributions on the presented antenna at $28 \mathrm{GHz}$. (a) $\mathrm{t}=0$, (b) $\mathrm{t}=\mathrm{T} / 4$, (c) $\mathrm{t}=\mathrm{T} / 2$, (d) $\mathrm{t}=3 \mathrm{~T} / 4$.

Therefore, the transmission magnitude and phase at the two wide slots can be calculated using (1) and (2). As a special case and also a readily realized method for the antenna design, the radiating resistance of the wide open slot can be designed as $100 \Omega$. The narrow slotline formed by the coupling between the patches can be designed with the characteristic impedance of $100 \Omega$ and transmission phase of $90^{\circ}$. Substituting these conditions into (1) and (2), one can get the following transmission coefficients,

$$
S_{21}=\frac{\sqrt{2}}{2} \quad \text { and } \quad S_{31}=-j \frac{\sqrt{2}}{2}
$$

It can be seen in (3) that equal magnitude and quadrature phase excitation can be achieved at two wide open slots. Finally, fed by two stair-shaped $50 \Omega$ microstrip lines, these two pairs of open slots, including open slots 1-2 and open slots 3-4, can be respectively and sequentially excited. Therefore, ideal CP radiation can be achieved for the presented differentially-fed antenna.

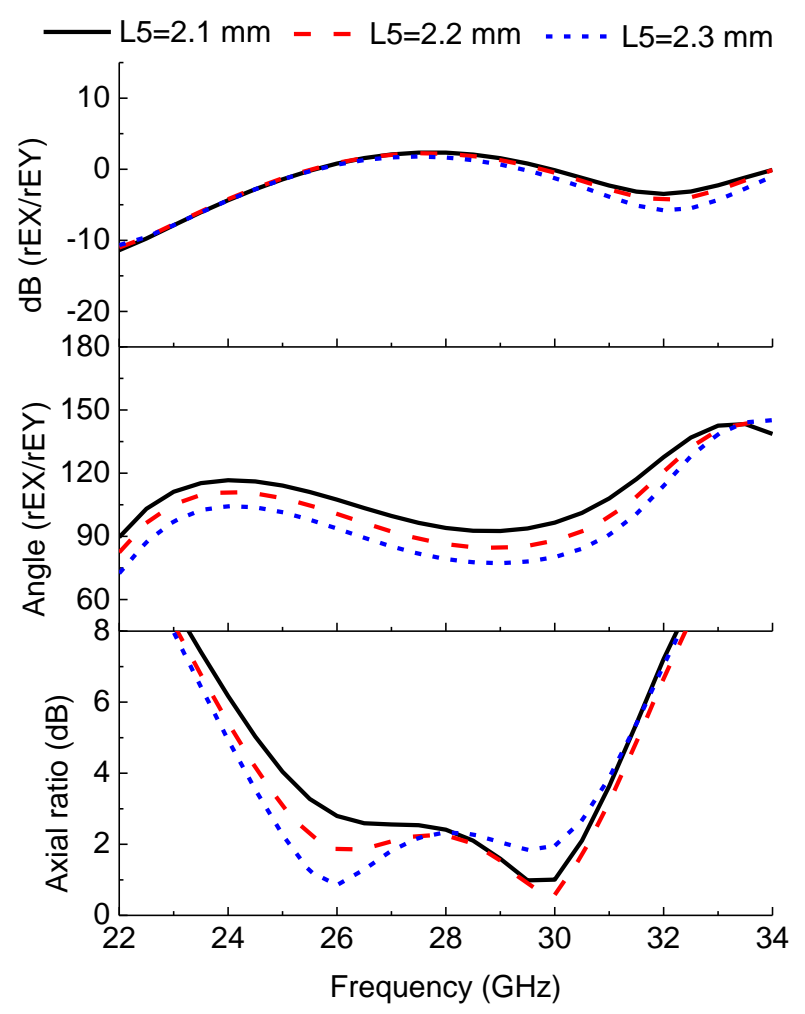

Fig. 4. Radiated far-field magnitude difference, phase difference, and axial ratio vary with different lengths of the slotline (L5).

To show the principle of $\mathrm{CP}$ radiation clearly, Fig. 3 gives the current distributions on the radiating patches at the different times. In the figure, $\mathrm{T}$ is the oscillation period at the frequency of $28 \mathrm{GHz}$, and auxiliary arrows are added to indicate the current directions. As can be seen in Fig. 3 (a), at the time $t=0$, the top and bottom wide open slots are firstly excited with strong current magnitude. After the time of a quarter period shown in Fig. 3 (b), strong current magnitude is observed on the left and right wide open slots. This denotes that, by using the inner center arranged slotline as the power transmission and phase shifter, an equal magnitude and quadrature phase excitation is obtained at the four wide open slots. Current distributions at the times of $\mathrm{t}=\mathrm{T} / 2$ and $\mathrm{t}=3 \mathrm{~T} / 4$ shown in Fig. 3 (c) and (d) further demonstrate that the four wide open slots can be 
sequentially excited with the $90^{\circ}$ phase delay. Therefore, based on the current distributions shown on the radiating patches, right-hand $\mathrm{CP}$ radiation is realized for the presented differentially-fed antenna.

Utilizing the equivalent circuit shown in Fig. 2, it also can be inferred that by changing the length of the narrow slotline, the phase difference between the far-field components $\mathrm{E}_{x}$ and $\mathrm{E}_{y}$ radiated by four wide open slots can be elaborately adjusted. Meanwhile, by changing the width of the narrow slotline, the characteristic impedance of the slotline can be changed. Accordingly, the magnitudes of the radiated far-field components $\mathrm{E}_{x}$ and $\mathrm{E}_{y}$ can be respectively adjusted. Therefore, the radiated $\mathrm{CP}$ performance can be easily adjusted by changing the parameters of the narrow slotline.

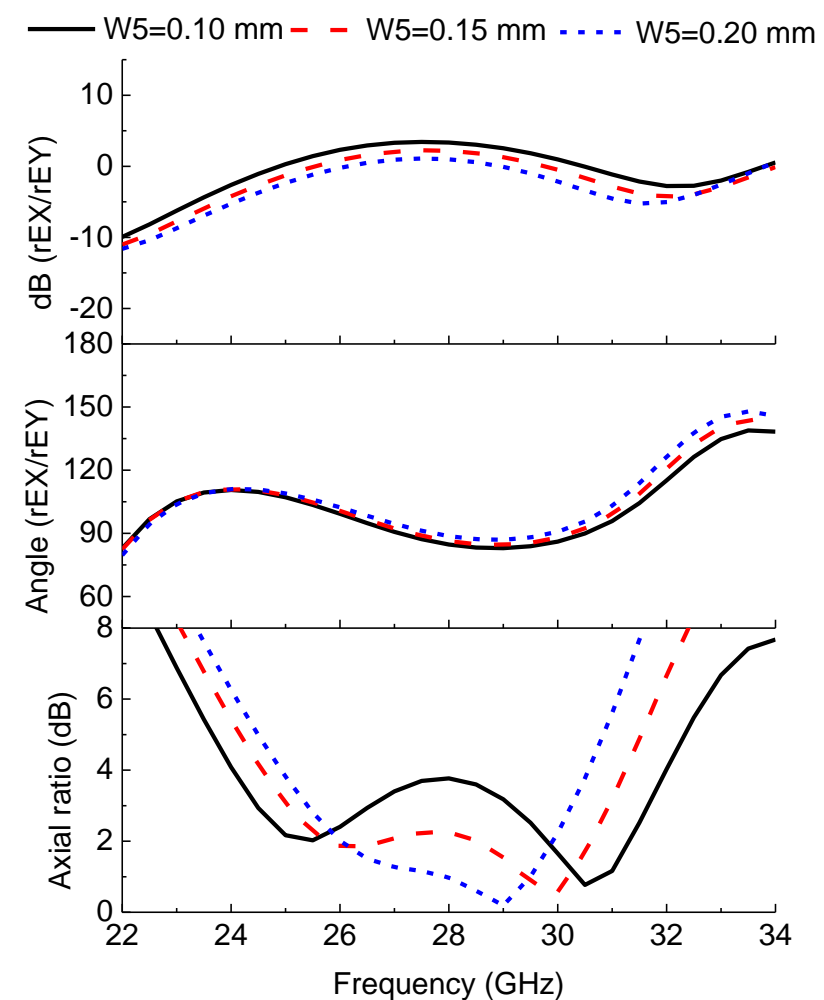

Fig. 5. Radiated far-field magnitude difference, phase difference, and axial ratio vary with different widths of the slotline (W5).

Fig. 4 shows the simulated far-field performance varies with different lengths of slotline (L5). As shown in the first two subfigures, when the length of the narrow slot is changed from $2.1 \mathrm{~mm}$ to $2.3 \mathrm{~mm}$, the phase difference between $\mathrm{E}_{x}$ and $\mathrm{E}_{y}$ is gradually increased. However, the magnitude difference between these two components is almost unchanged. This denotes that by changing the length of the narrow slot, the radiated phase difference of the two orthogonal components can be elaborately controlled, while the radiated magnitude difference is nearly unaffected. The bottom subfigure shows the related response of the resulted AR. It can be seen that the resulted AR is changed a lot at the lower frequency band, compared to the AR at the upper frequency band as the increase of L5. While the AR at the center frequency $28 \mathrm{GHz}$ is nearly unchanged. This is because the magnitude difference between the radiated $\mathrm{E}_{x}$ and $\mathrm{E}_{y}$ at the upper frequency band is more stable and closer to $0 \mathrm{~dB}$ as compared to the lower frequency band.

Fig. 5 shows the simulated far-field performance varies with different widths of the narrow slot (W5). It can be observed in the top subfigure, as the width becomes wider, more energy is radiated in the $y$-axis direction, and the magnitude ratio of $\mathrm{E}_{x} / \mathrm{E}_{y}$ is reduced. Therefore, this parameter can be primarily used to adjust the radiated magnitudes of each far-field components. It should also be noted that the phase difference between the two components is only slightly changed in the center subfigure. This slight change is mainly caused by the approximate equivalence of the slotline transmission line. As the increase of the slot width, the center reference ground plane shared by the two slotlines is reduced. Therefore, the distributed inductance of the slot transmission line is increased, and the phase difference between the two orthogonal components is changed accordingly. The bottom subfigure shows the resulted AR as the increase of W5. It can be seen that, different from the length of the slotline, the width of the narrow slot can have a significant effect on the AR at the center frequency. This makes the resulted AR being improved at the center frequency as the increase of W5. However, the total AR bandwidth will be narrowed as the increase of the slot width, as indicated by the magnitude and phase variances in the top subfigure.

\section{Antenna Study}

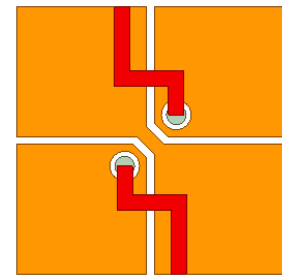

Antenna 1

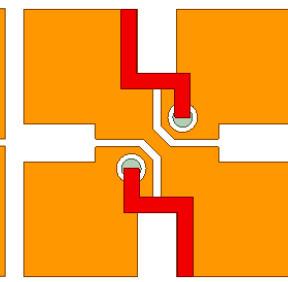

Antenna 2

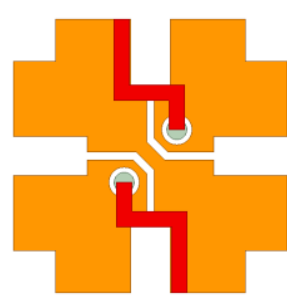

Antenna 3
Fig. 6. Evolution process of the different antennas, Antenna 3 is the proposed antenna.

In the design of the proposed antenna, the slots are classified into two types, the transmission section and the radiation section. Owing to this elaborate configuration, the proposed antenna can have both wide AR and impedance bandwidths. Fig. 6 shows the evolution process of the proposed antenna. As shown in the figure, only narrow slots are etched on the patches of Antenna 1. In Antenna 2, wide open slots are utilized to improve the $\mathrm{CP}$ radiation. To further improve the radiation and impedance characteristics, square cuts are etched on each corner of the patches on Antenna 3. It should be noted that Antenna 3 is the proposed antenna in this work.

Fig. 7 shows the simulated ARs and reflection coefficients of these different evolution antennas. As can be seen in Fig. 7 (a), although Antenna 1 can have two AR valleys from $22 \mathrm{GHz}$ to $34 \mathrm{GHz}$, each of them is far away from each other with high AR values, which represents the low $\mathrm{CP}$ radiation efficiency. After evolving the ending narrow slot into a wide slot as Antenna 2, the $\mathrm{CP}$ radiation is improved, especially for the second AR zero. It can be seen that the axial ratio at the upper band is improved significantly. However, the AR at the lower band is still very high. When four square cuts are etched on the patches for Antenna 3, both the first and the second AR zeros move closer 
to the center, and a wide AR bandwidth of less than $3 \mathrm{~dB}$ from $25.1 \mathrm{GHz}$ to $30.9 \mathrm{GHz}$ is obtained.

Fig. 7 (b) shows the corresponding simulated reflection coefficients of these different evolution antennas. It can be observed that Antenna 1 has high reflection at the input port, which is mainly caused by the low radiation efficiency of the narrow slotline at the end. As the narrow slotline at the end is changed into wide open slot for effective radiation, the reflection at the upper band is reduced for Antenna 2. To further improve the impedance at the lower band, square cuts are etched on the patches for Antenna 3. Therefore, the reflection zero at the lower band moves to the center, and the impedance bandwidth for $\mathrm{S}_{\mathrm{dd} 11}<-10 \mathrm{~dB}$ is enhanced to $24.9-33 \mathrm{GHz}$.

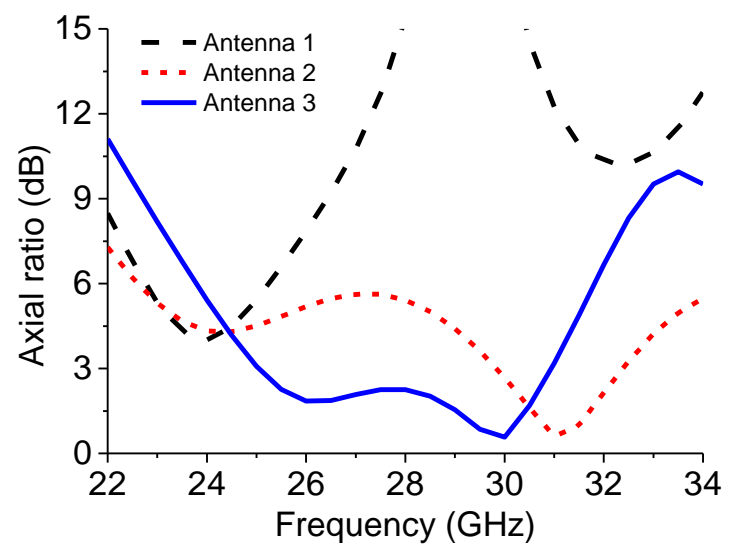

(a)

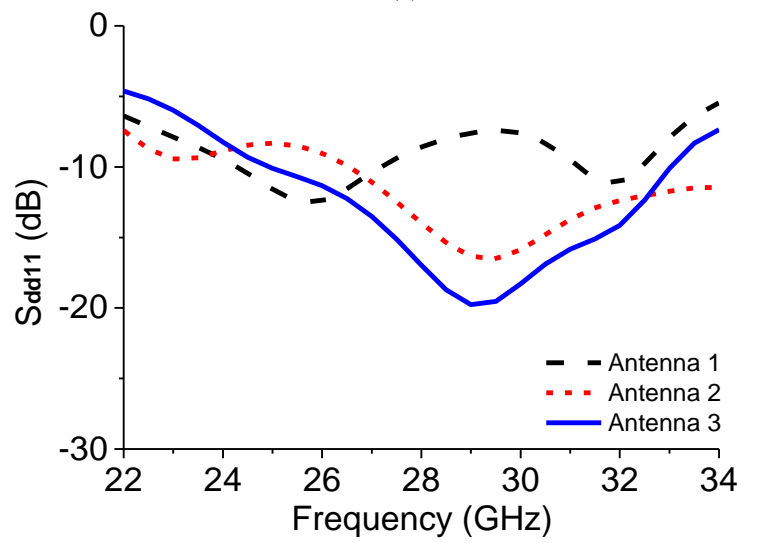

(b)

Fig. 7. (a) Axial ratio and (b) reflection coefficient of the different evolution antennas.

In the design of the proposed antenna, parameters of the length of wide open slot (L4), the width of the square cut on the patch (W4), and the length of the feed line (L1) can also have a significant effect on performances of the impedance and AR. Fig. 8 shows the detailed effects of these parameters. As shown in Fig. 8 (a), when the length of the wide open slot (L4) is increased from $1.2 \mathrm{~mm}$ to $1.6 \mathrm{~mm}$, both the impedance and AR bandwidths are shifted to the lower frequency. This denotes that the length of wide open slot works as the basic resonance for the presented antenna. By changing this parameter, the whole bandwidth can be changed accordingly.

Fig. 8 (b) shows the effect of the width of the square cut on the patch. It can be seen that, as the increase of W4, the AR at the upper frequency band is changed substantially. The impedance bandwidth for $\mathrm{S}_{\mathrm{dd} 11}<-10 \mathrm{~dB}$ and $\mathrm{AR}$ at the lower band are only slightly moved. This means that the square cut can be mainly used to improve the $\mathrm{CP}$ radiation at the upper frequency band. But it should be noted that this parameter also has a slight influence on the impedance bandwidth.

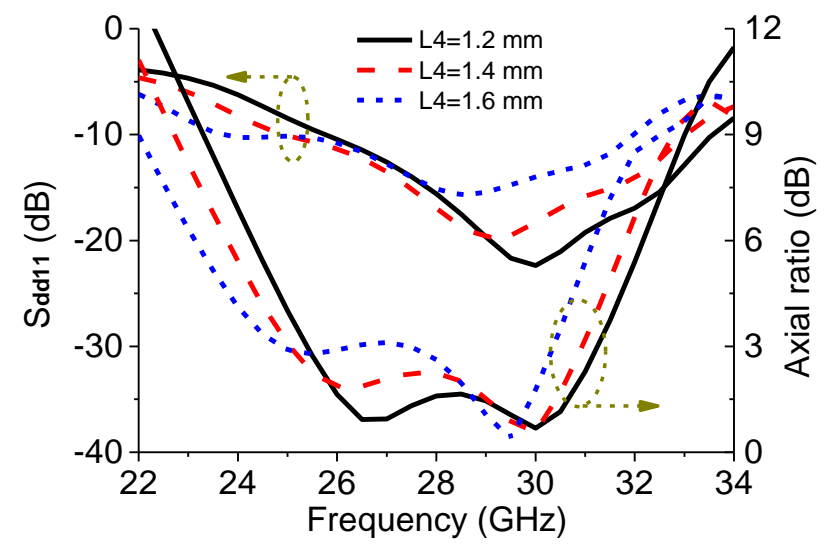

(a)

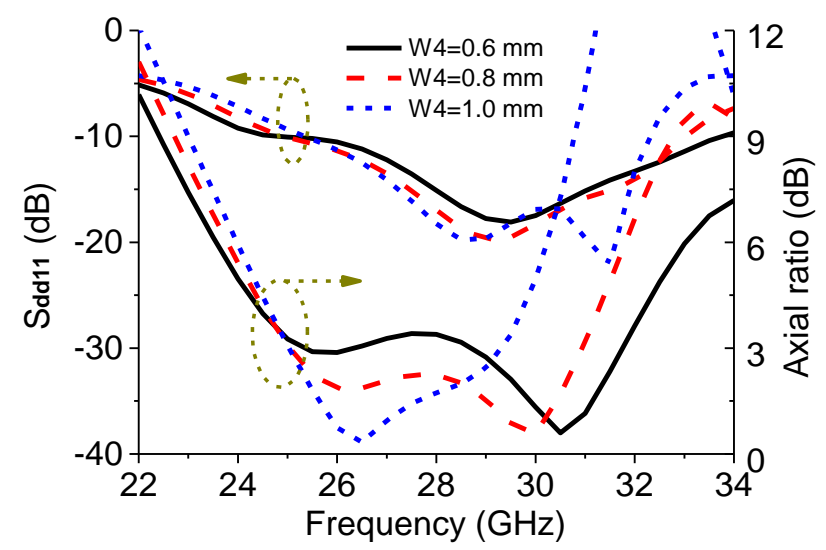

(b)

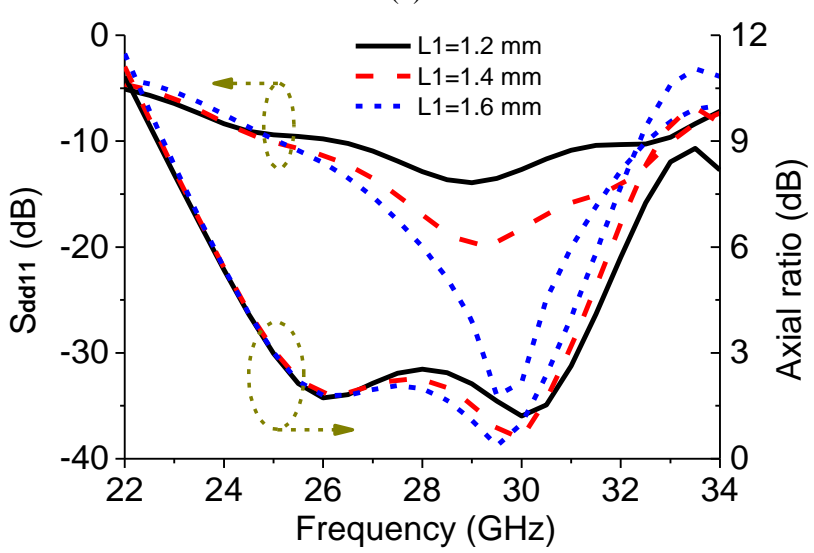

(c)

Fig. 8. Simulated reflection coefficient and AR vary with different parameters of the proposed CP antenna. (a) The length of wide open slot (L4). (b) The width of the square cut on the patch (W4). (c) The length of the feed line (L1).

Fig. 8 (c) shows the effect of the length of the microstrip feed line on the performance of the antenna. As shown in the figure, when $\mathrm{L} 1$ is increased from $1.2 \mathrm{~mm}$ to $1.6 \mathrm{~mm}$, the reflection at the upper frequency band is improved a lot with deep reflection zero, and the AR at the upper frequency band is only slightly moved to lower frequency. The reflection coefficient and AR at the lower frequency band are almost unchanged as the variance 
of L1. Therefore, based on the study of this parameter, L1 can be used to improve the impedance bandwidth at the upper frequency band, while it only has slight effect on the AR at the upper frequency band.

\section{Results and Discussion}

The proposed CP antenna element was first fabricated and measured for performance verification. To preserve the copper surface from oxidation and contamination, a standard surface treatment method of organic solderability preservative (OSP) is used in antenna fabrication. Fig. 9 shows the fabricated prototype of the proposed antenna. The S-parameters and radiation patterns were measured by the Anritsu 37397C vector network analyser and the ASYSOL far-field antenna measurement system at the University of Kent. Fig. 10 shows the simulated and measured reflection coefficients and ARs of the fabricated antenna. It can be seen that the measured impedance bandwidth for $S_{d d 11}<-10 \mathrm{~dB}$ is from $24.5 \mathrm{GHz}$ to $31.4 \mathrm{GHz}$, which is slightly narrower than the simulated results. This difference in impedance bandwidths is probably caused by the assembly and soldering error in the fabrication. The measured CP bandwidth for $\mathrm{AR}<3 \mathrm{~dB}$ is from $25.5 \mathrm{GHz}$ to 31.3 $\mathrm{GHz}$, which has a slight shift to the upper frequency.

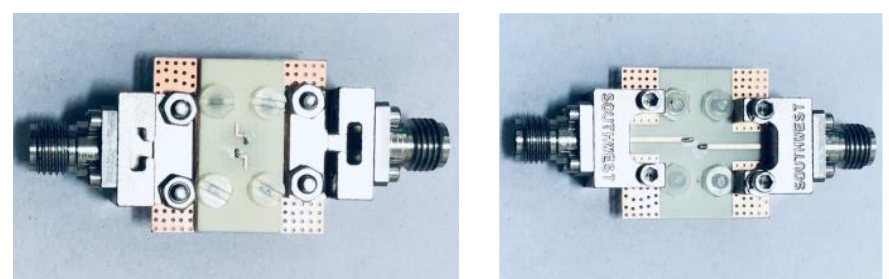

Fig. 9. Fabricated prototype of the proposed CP antenna.

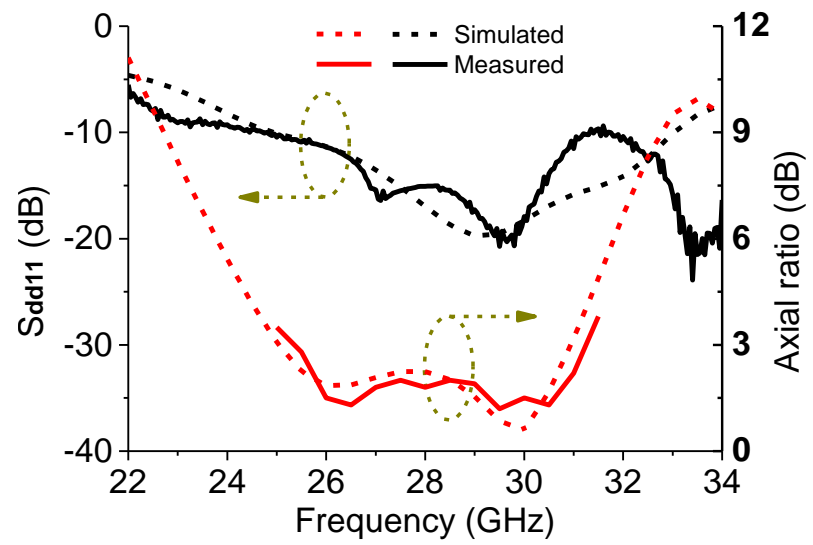

Fig. 10. Measured and simulated S-parameters and ARs of the fabricated CP antenna element.

The measured and simulated normalized radiation patterns in xz-plane and yz-plane at $26 \mathrm{GHz}, 28 \mathrm{GHz}$, and $30 \mathrm{GHz}$ are shown in Fig. 11. In the figure, right-hand $\mathrm{CP}$ radiation patterns are the co-polarization, and left-hand $\mathrm{CP}$ patterns are the cross-polarization. It can be seen that the presented CP antenna have a good symmetry in its radiation patterns, and the measured radiation patterns agree well with the simulated results. The measured half-power beamwidth (HPBW) is $64-69^{\circ}$ in xz-plane and $60-81^{\circ}$ in yz-plane. The measured $3 \mathrm{~dB}$ AR beamwidth (ARBW) is $60-76^{\circ}$ in xz-plane, and $51-70^{\circ}$ in yz-plane. The measured AR within HPBW is lower than $4.3 \mathrm{~dB}$ in xz-plane, and lower than $4.5 \mathrm{~dB}$ in yz-plane within the operation bandwidth. The measured front to back ratio is higher than $14.9 \mathrm{~dB}$ within the bandwidth. The slight discrepancies between the measured and simulated co-polarized and cross-polarized radiation patterns could be caused by the manufacture tolerances, the measurement errors in the chamber, and the instability of the substrates, etc.

Fig. 12 shows the measured peak realized gain of the presented antenna, which is also compared with the simulated result. As shown in the figure, the measured gain is slightly lower than the simulated value, which is varying from $6.5 \mathrm{dBic}$ to 8 dBic. The decreasing gain is mainly caused by the relatively large ground size for the convenience of the connection to the end-launch SMK connectors and being less affected by the connectors. This phenomenon can be avoided by increasing the number of antenna elements for large array designs.
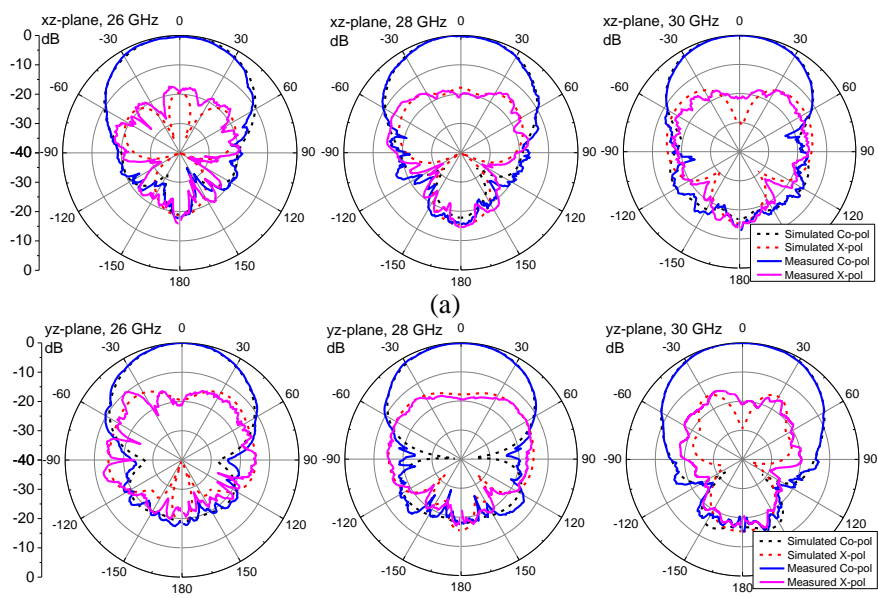

(b)

Fig. 11. Measured and simulated radiation patterns of the fabricated $\mathrm{CP}$ antenna element at different frequencies in (a) xz-plane and (b) yz-plane.

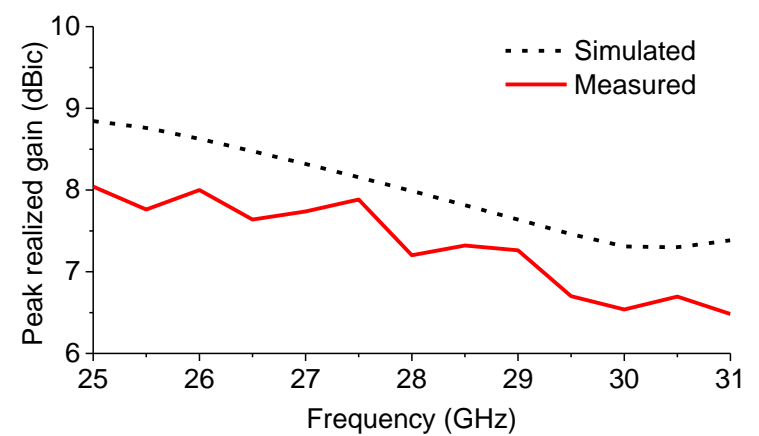

Fig. 12. Measured and simulated peak realized gain of the fabricated $\mathrm{CP}$ antenna element.

\section{III. $1 \times 4$ LINEAR ARRAY}

\section{A. Linear Array Design}

The presented differentially-fed $\mathrm{CP}$ antenna element was then developed into a $1 \times 4$ linear array, and its configuration is shown in Fig. 13. The distance between each element is designed as $7 \mathrm{~mm}$, which equals $0.65 \lambda_{0}$ (where $\lambda_{0}$ is the free space wavelength at $28 \mathrm{GHz}$ ). Four presented antenna elements are fed by four-way T-typed power divider with equal magnitude and phase excitation for the broadside radiation. Note that ports $P_{+}$and $P_{-}$shown in the top and bottom of the figure are the differential port-pair of the linear array. To 
further broaden the beam coverage of the $1 \times 4$ linear array, another two feed networks with different fixed phase delays are used to verify the beam scanning performance of the linear array. The feed networks with fixed phase delay are shown in Fig. 14, which are utilized to realize another two beams at the $\theta$-directions of $-18^{\circ}$ and $18^{\circ}$. Then, the total half-power beam coverage can be enhanced up to $-27.5^{\circ}$ to $27.5^{\circ}$.

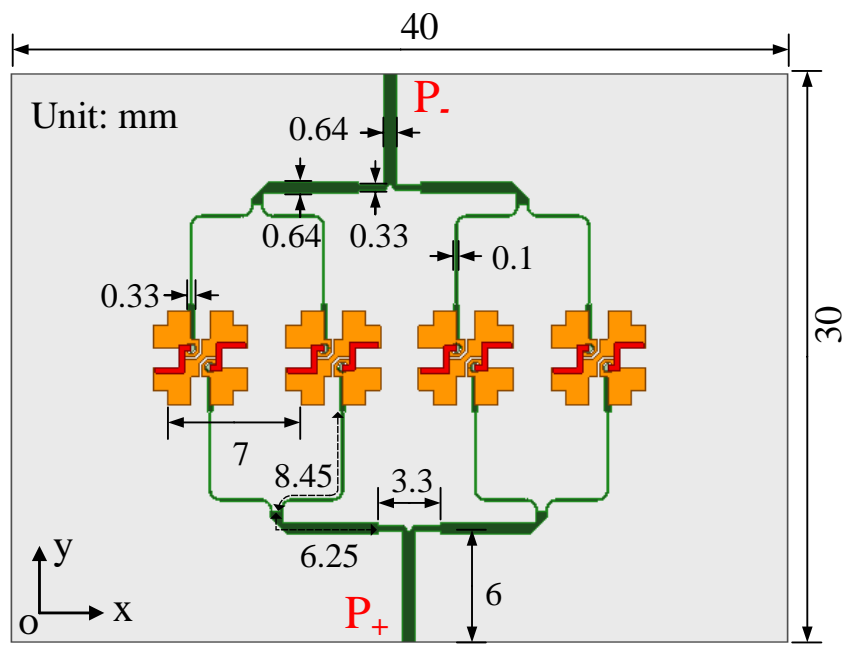

Fig. 13. Configuration of the designed $1 \times 4$ linear array.

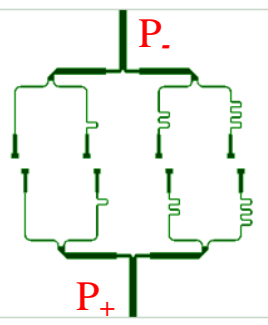

(a)

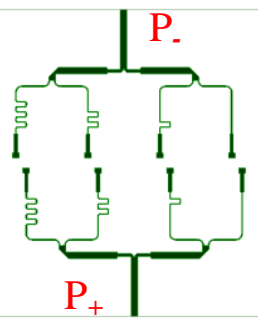

(b)
Fig. 14. Different feed networks for beam scanning performance verification. (a) Feed network for scan angle of $18^{\circ}$ and (b) feed network for scan angle of $-18^{\circ}$.

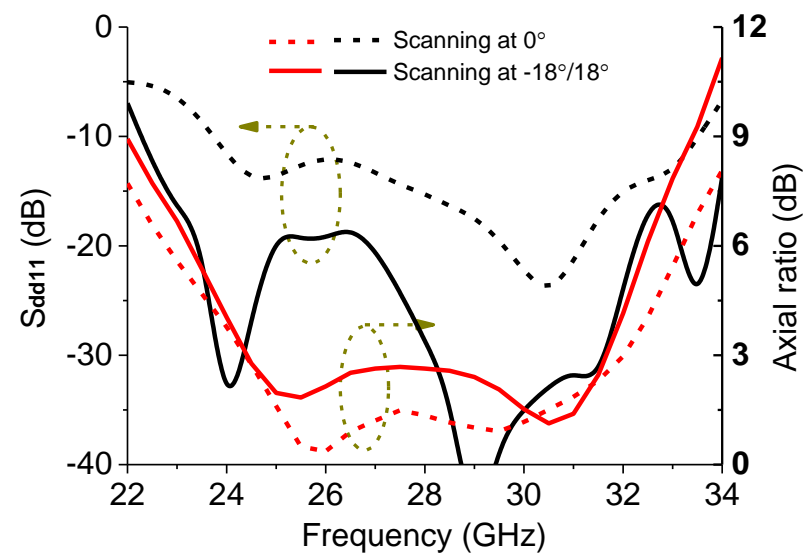

Fig. 15. Simulated reflection coefficients and ARs under different scan angles.

Fig. 15 shows the simulated reflection coefficients and ARs when scanning to different angles. Because of the symmetry of the feed network, simulated results for the beam scan angle at $-18^{\circ}$ are almost the same as the results at $18^{\circ}$, so only one is shown for brevity. As shown in the figure, the impedance bandwidth for $S_{\text {dd11 }}<-10 \mathrm{~dB}$ is $24.0-33.5 \mathrm{GHz}$ when the beam scans to the broadside direction, and the bandwidth for $\mathrm{AR}<3$ $\mathrm{dB}$ is $24.4-32.0 \mathrm{GHz}$. When the antenna beam scans to $18^{\circ} /-18^{\circ}$, a much lower reflection coefficient is obtained with the impedance bandwidth covering 22.4-34.0 GHz. The simulated bandwidth for $\mathrm{AR}<3 \mathrm{~dB}$ is slightly deteriorated and reduced to 24.4-31.6 GHz. Fig. 16 shows the simulated radiation patterns at two edge frequencies of $25 \mathrm{GHz}$ and $31 \mathrm{GHz}$ when beam scanning to $-18^{\circ}, 0^{\circ}$, and $18^{\circ}$. The simulated radiation patterns show that the overall half-power beamwidth of these three beams can cover the beam range within $55^{\circ}$, and the simulated $\mathrm{AR}$ in each scanning direction is less than $3 \mathrm{~dB}$. When the beam scanning to $18^{\circ}$, the sidelobe at $31 \mathrm{GHz}$ is slightly increased compared to the sidelobe at $25 \mathrm{GHz}$. But it is still acceptable, and its level is $10.6 \mathrm{~dB}$ lower than the mainlobe.

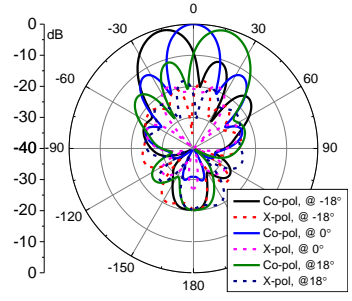

(a)

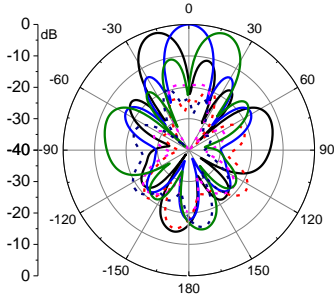

(b)
Fig. 16. Simulated radiation patterns with the scan angles at $-18^{\circ}, 0^{\circ}$, and $-18^{\circ}$ at the frequencies of (a) $25 \mathrm{GHz}$ and (b) $31 \mathrm{GHz}$.

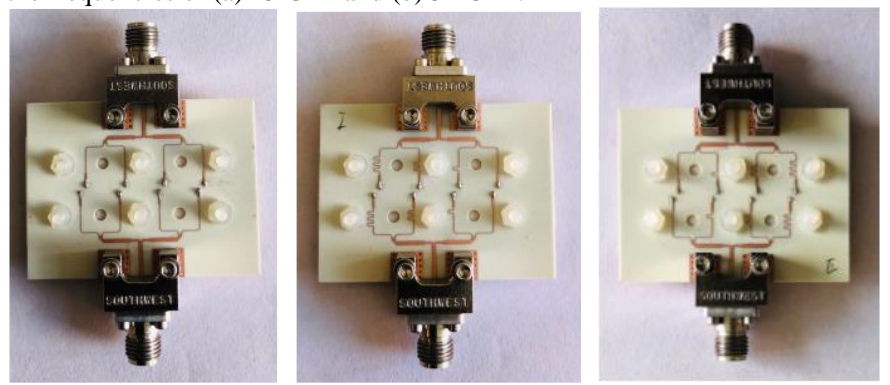

Fig. 17. Fabricated prototype of the proposed linear arrays with beam scanning performance.

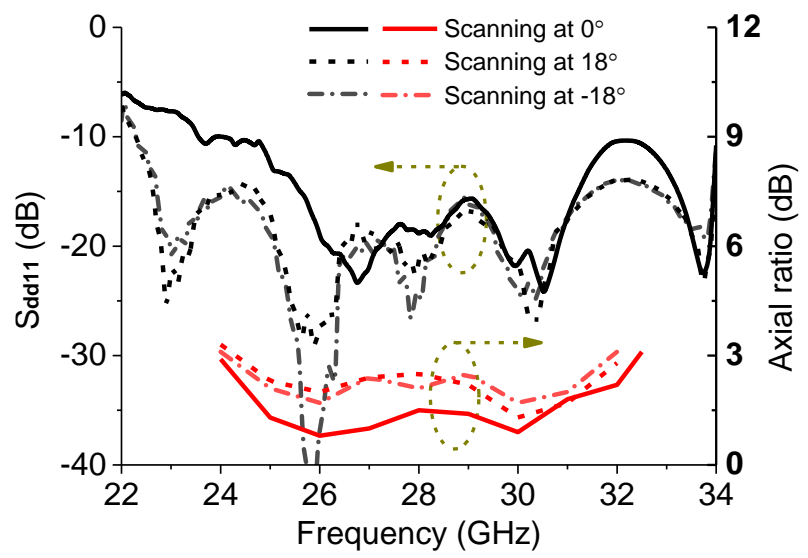

Fig. 18. Measured reflection coefficients and ARs of the fabricated $1 \times 4$ linear arrays.

\section{B. Results and Discussion}

This $1 \times 4$ linear array with beam scanning performance was fabricated and measured at the University of Kent. Fig. 17 shows three fabricated $1 \times 4$ linear arrays with different phase-delay feed networks. S-parameters and ARs of the linear array fed by different feed networks were measured, and these results are shown in Fig. 18. As expected from the simulated results, when the linear array scans to the broadside direction, 
its measured impedance bandwidth for $S_{d d 11}<-10 \mathrm{~dB}$ is $23.5-34$ $\mathrm{GHz}$. When scanning to $18 /-18^{\circ}$, its measured impedance bandwidth is slightly wider and covers the frequency band of 22.3-34 GHz. The measured $\mathrm{CP}$ bandwidth of $\mathrm{AR}<3 \mathrm{~dB}$ when scanning to the broadside direction is $23.8-32.4 \mathrm{GHz}$, while the CP bandwidth when scanning to $18 /-18^{\circ}$ is slightly reduced to 24.1-31.9 GHz. Compared to the simulated results shown in Fig. 15 , the measured impedance and AR bandwidths are slightly shifted to upper frequency, but these results agree well with each other.

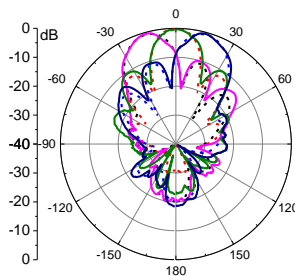

(a)
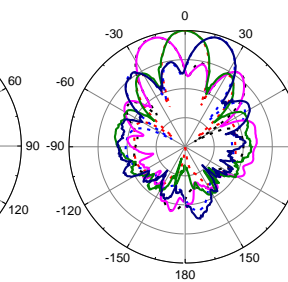

(b)

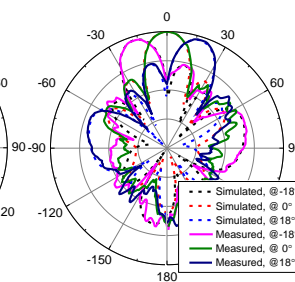

(c)
Fig. 19. Measured normalized beam scanning performance of the linear arrays at (a) $25 \mathrm{GHz}$, (b) $28 \mathrm{GHz}$, and (c) $31 \mathrm{GHz}$.

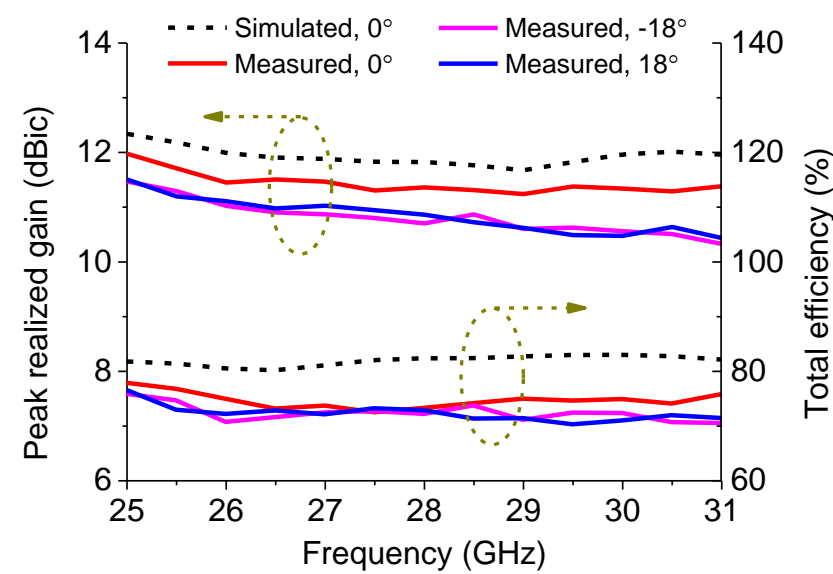

Fig. 20. Measured and simulated peak realized gains and total efficiencies of the fabricated linear arrays.

The measured radiation patterns at different operation frequencies with beam scanning performance are shown in Fig. 19. It can be seen that the measured radiation patterns agree well with the simulated radiation patterns. In xz-plane, when the developed linear array antenna scans to the broadside direction, the measured HPBW is $18-19^{\circ}$ from $25 \mathrm{GHz}$ to 31 GHz. The measured $3 \mathrm{~dB}$ ARBW is $24-30^{\circ}$, which is wider than the HPBW. So the radiated AR within HPBW is less than $3 \mathrm{~dB}$ within the bandwidth. In yz-plane, the measured HPBW and $3 \mathrm{~dB}$ ARBW are very similar to the antenna element, which are $66-81^{\circ}$ and $55-75^{\circ}$, and the measured AR is less than $4.3 \mathrm{~dB}$ within the HPBW. When it scans to $18^{\circ} / 18^{\circ}$, the measured half-power beamwidths are $20^{\circ}$ and $19^{\circ}$ at $25 \mathrm{GHz}$ and $31 \mathrm{GHz}$. The measured total half-power beam coverage is ranging from $-27^{\circ}$ to $27^{\circ}$. The scan losses are $0.6 \mathrm{~dB}$ and $1.2 \mathrm{~dB}$ for the beams scanning to $18^{\circ} / 18^{\circ}$ at $25 \mathrm{GHz}$ and $31 \mathrm{GHz}$. The measured sidelobes are $9.6 \mathrm{~dB}$ lower than the mainlobe when the beam scans to $18^{\circ} \% 18^{\circ}$.

Fig. 20 compares the measured peak realized gain and total efficiency of the antenna array when scanning to different angles. It can be seen that the measured peak realized gain varies from $11.2 \mathrm{dBic}$ to $12 \mathrm{dBic}$ for the broadside beam. When the beam scans to $18^{\circ} /-18^{\circ}$, the peak realized gain is slightly reduced from $11.5 \mathrm{dBic}$ to $10.3 \mathrm{dBic}$ within the bandwidth. The measured total efficiency (the calculation includes the impedance mismatch loss) is above $72.5 \%$ for the broadside beam, and above $70.3 \%$ when the beam scans to $18 \%-18^{\circ}$.

\section{IV. $4 \times 4$ PLANAR ARRAY}

\section{A. Planar Array Design}

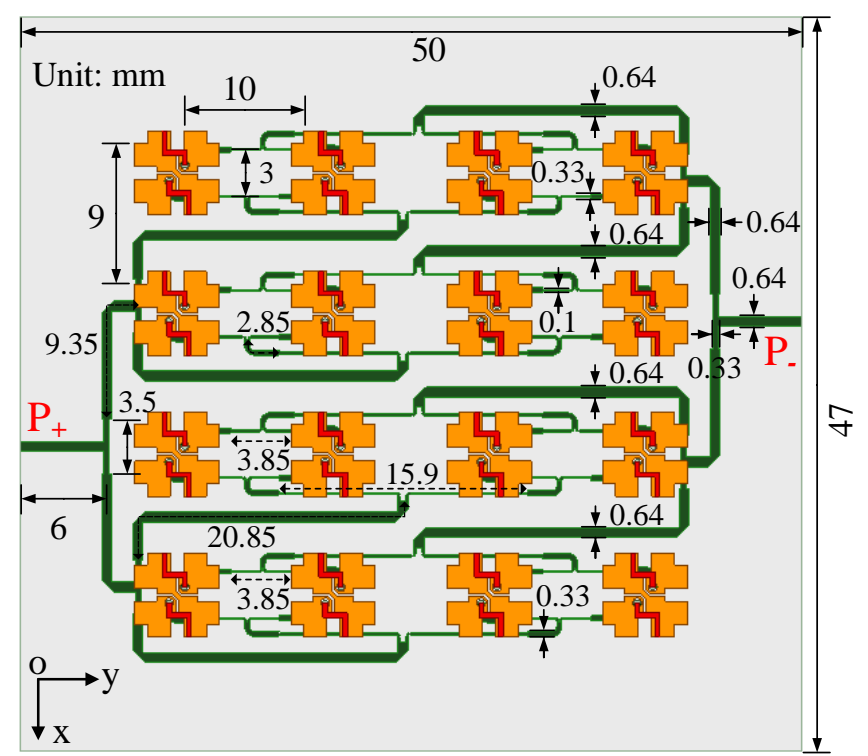

Fig. 21. Configuration of the designed $4 \times 4$ linear array.
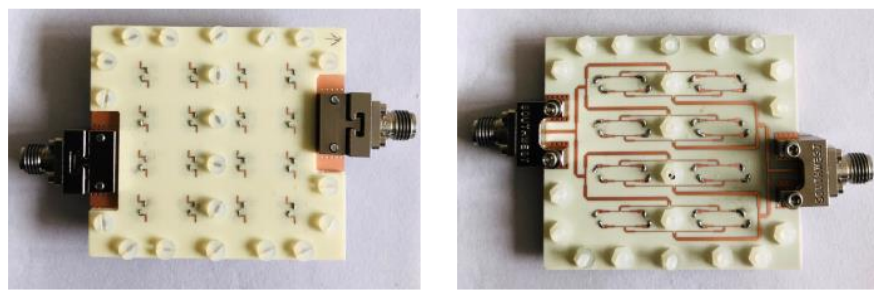

Fig. 22. Photographs of the fabricated $4 \times 4$ planar array.

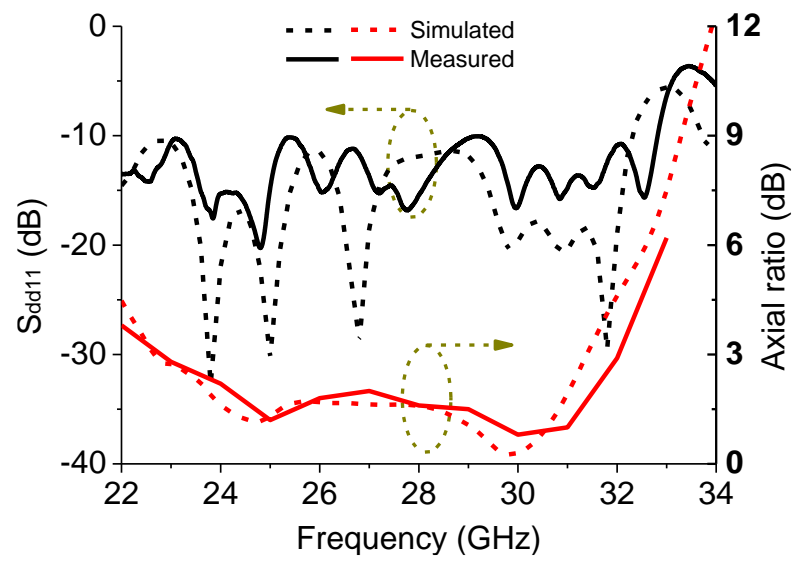

Fig. 23. Measured and simulated reflection coefficients and ARs of the fabricated $4 \times 4$ array.

To realize high gain and stable unidirectional $\mathrm{CP}$ radiation, another $4 \times 4$ planar array is designed and its configuration is shown in Fig. 21. The element distance is slightly increased compared to the linear array in Section III for high gain 
radiation. The element distance is $9 \mathrm{~mm}\left(0.84 \lambda_{0}\right)$ in $\mathrm{x}$-axis direction, and $10 \mathrm{~mm}\left(0.93 \lambda_{0}\right)$ in y-axis direction, (where $\lambda_{0}$ is the free space wavelength at $28 \mathrm{GHz}$ ). The planar array is fed by two T-typed power dividers for differential excitation. Ports $P_{+}$and $P_{-}$shown in the left and right of the figure are the differential port pair of the planar array.

\section{B. Results and Discussion}
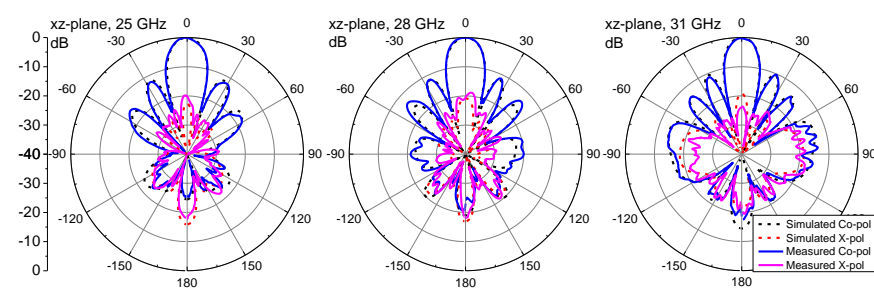

(a)
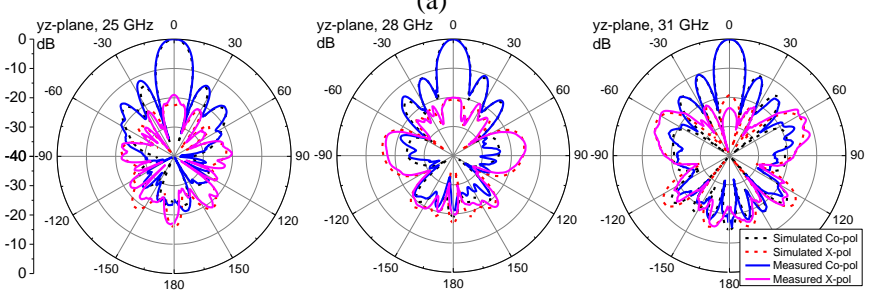

(b)

Fig. 24. Measured and simulated radiation patterns of the fabricated $4 \times 4$ planar array at different frequencies in (a) xz-plane and (b) yz-plane.

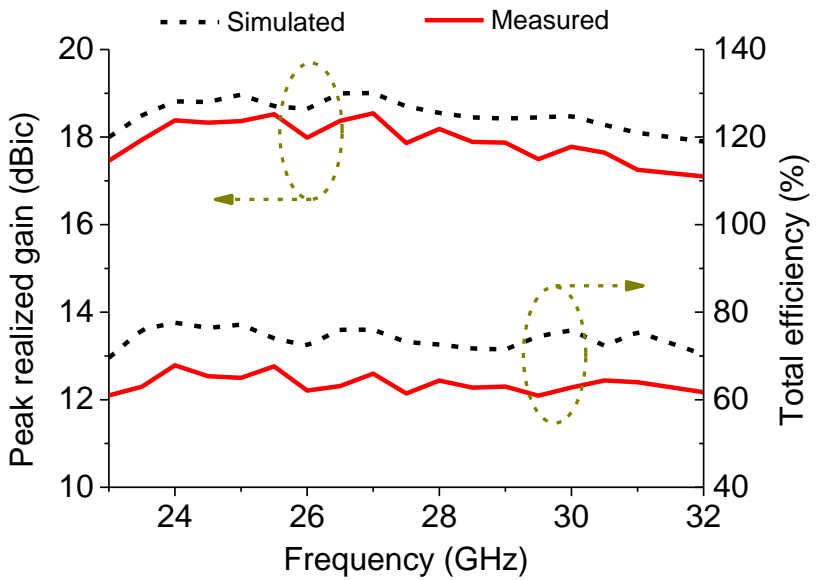

Fig. 25. Measured and simulated peak realized gains and total efficiencies of the fabricated $4 \times 4$ planar array.

This $4 \times 4$ planar array was designed and measured for performance verification. Fig. 22 shows the photographs of the fabricated array prototype. The measured reflection coefficient and AR of this planar array are shown in Fig. 23. It can be seen that the measured impedance bandwidth for $S_{d d 11}<-10 \mathrm{~dB}$ is 22.0-32.8 GHz. The measured $\mathrm{CP}$ bandwidth for $\mathrm{AR}<3 \mathrm{~dB}$ is 22.8-32.0 GHz. Both the impedance and AR bandwidths have a slight shift to the upper frequency compared to the simulated results, but still have a good agreement. This slight discrepancy could be caused by the manufacture, assembly, and soldering errors in the array fabrication.

The measured normalized radiation patterns at $25 \mathrm{GHz}, 28$ $\mathrm{GHz}$, and $31 \mathrm{GHz}$ are shown in Fig. 24, which are also compared with the simulated results. As can be seen, a good agreement is obtained between the simulated and measured radiation patterns. The measured HPBW of the planar array is $15-17^{\circ}$ in xz-plane and $14-16^{\circ}$ in yz-plane within the bandwidth. The measured $3 \mathrm{~dB}$ ARBW is $28-30^{\circ}$ in xz-plane and $19-21^{\circ}$ in yz-plane, which can ensure that the radiated AR within the HPBW is less than $3 \mathrm{~dB}$. The measured front to back ratio is higher than $16.7 \mathrm{~dB}$ within the bandwidth.

Fig. 25 gives the measured peak realized $\mathrm{CP}$ gain and total efficiency. Compared to the simulated realized gain, the measured result is slightly lower than the simulated value. The measured peak realized gain is very stable and varies between $17.1 \mathrm{dBic}$ and $18.5 \mathrm{dBic}$. Because of the increased number of antenna elements and increased length of the feed network, the total efficiency is slightly reduced compared to the linear array. Despite that, it is still very good and better than $60.9 \%$ within the bandwidth.

\section{Loss Evaluation and Performance Comparison}

The gain and efficiency losses of the developed arrays are mainly from the conduction loss, dielectric loss, and impedance mismatch loss. To further evaluate these, insertion losses on the different feed networks of the arrays and SMK end-launch connectors are listed in Table I. It can be seen that, the simulated losses on microstrip-line based feed networks become obvious in mmWave frequency band. As the increase of the frequency, more losses can be introduced, this is also the reason why relatively low gain and efficiency are achieved at the upper frequency. To pursuit lower loss and higher efficiency, a low-loss feed network should be considered, such as using waveguide or substrate integrated waveguide.

TABLE I

\begin{tabular}{|c|c|c|c|c|}
\hline \multirow{2}{*}{ Frequency } & \multicolumn{2}{|c|}{${ }^{1} 1 \times 4$ linear array } & \multirow{2}{*}{$\begin{array}{c}{ }^{1} 4 \times 4 \text { planar } \\
\text { array }\end{array}$} & \multirow{2}{*}{$\begin{array}{l}{ }^{2} \text { End-launch } \\
\text { connector }\end{array}$} \\
\hline & $0^{\circ}$ & $+/-18^{\circ}$ & & \\
\hline $25 \mathrm{GHz}$ & $0.71 \mathrm{~dB}$ & $0.71 \mathrm{~dB}$ & $1.2 \mathrm{~dB}$ & $0.30 \mathrm{~dB}$ \\
\hline $28 \mathrm{GHz}$ & $0.74 \mathrm{~dB}$ & $0.80 \mathrm{~dB}$ & $1.45 \mathrm{~dB}$ & $0.32 \mathrm{~dB}$ \\
\hline $31 \mathrm{GHz}$ & $0.85 \mathrm{~dB}$ & $0.95 \mathrm{~dB}$ & $1.75 \mathrm{~dB}$ & $0.33 \mathrm{~dB}$ \\
\hline
\end{tabular}

Table II compares the recently published differentially-fed $\mathrm{CP}$ antennas for mmWave applications, where $\lambda_{0}$ is the free space wavelength at the central operating frequency. To ensure good CP performance, it is difficult for $\mathrm{CP}$ antennas realizing wide beam scanning ranges. The $\mathrm{CP}$ antenna in [14] is a $1 \times 4$ linear array antenna, which can scan beams ranging from $-25^{\circ}$ to $25^{\circ}$. However, due to the limitation of its antenna size, the element distance in the linear array is $0.75 \lambda_{0}$. In addition, when the beam scans to $25^{\circ}$, the measured sidelobes are only $6 \mathrm{~dB}$ lower than the mainlobe, and the resulted ARs of each scan beam are even not given in the article. Compared to this work, although the proposed $1 \times 4$ linear array can scan from $-18^{\circ}$ to $18^{\circ}$ with the total beam coverage from $-27^{\circ}$ to $27^{\circ}$, its measured sidelobes keep around $10 \mathrm{~dB}$ lower than the mainlobe, and the resulted $\mathrm{AR}$ of each beam is less than $3 \mathrm{~dB}$. In addition, our proposed linear array keeps low profile, but owns wider overlapped impedance and AR bandwidth. [20], [22], and [23] are the recently developed differentially-fed array antennas. Compared to these arrays with similar relative thickness, our presented $4 \times 4$ planar array keeps simple layer configuration and low profile, but has a wider impedance and AR bandwidth. 
TABLE II

DETAILED COMPARISON OF THE RECENTLY PUBLISHED DiFFERENTIALly-Fed CP ANTENNAS FOR MMWAVE APPLICATIONS

\begin{tabular}{|c|c|c|c|c|c|c|c|c|c|c|c|c|c|c|c|}
\hline \multirow[b]{2}{*}{ Ref. } & \multirow[b]{2}{*}{$\begin{array}{c}\mathrm{S}_{11} \\
(\mathrm{~dB})\end{array}$} & \multirow[b]{2}{*}{$\begin{array}{c}\text { AR } \\
(\mathrm{dB})\end{array}$} & \multirow[b]{2}{*}{$\begin{array}{c}\text { Overlapped } \\
\text { BW (GHz) }\end{array}$} & \multirow[b]{2}{*}{ RBW } & \multirow[b]{2}{*}{$\begin{array}{c}\text { Thick- } \\
\text { ness }\end{array}$} & \multirow[b]{2}{*}{$\begin{array}{l}\text { Copper } \\
\text { Layers }\end{array}$} & \multirow[b]{2}{*}{$\begin{array}{l}\text { Gain } \\
(\mathrm{dBic})\end{array}$} & \multirow[b]{2}{*}{$\begin{array}{l}\eta_{T} \\
(\%)\end{array}$} & \multicolumn{3}{|c|}{ xz-plane } & \multicolumn{3}{|c|}{ yz-plane } & \multirow[b]{2}{*}{$\begin{array}{c}\text { Scanning } \\
\text { Range } \\
\left(^{\circ}\right)\end{array}$} \\
\hline & & & & & & & & & $\begin{array}{c}\text { HPBW } \\
\left(^{\circ}\right)\end{array}$ & $\begin{array}{c}3 \mathrm{~dB} \\
\mathrm{ARBW} \\
\left({ }^{\circ}\right)\end{array}$ & $\begin{array}{c}\text { AR } \\
\text { within } \\
\text { HPBW }\end{array}$ & $\begin{array}{c}\text { HPBW } \\
\left(^{\circ}\right)\end{array}$ & $\begin{array}{c}3 \mathrm{~dB} \\
\mathrm{ARBW} \\
\left(^{\circ}\right)\end{array}$ & $\begin{array}{c}\text { AR } \\
\text { within } \\
\text { HPBW }\end{array}$ & \\
\hline [14] & $<-10$ & $<3$ & $33.5-42$ & $22.5 \%$ & $0.38 \lambda_{0}$ & 3 & $10.4-12.8$ & $>51.6$ & NG & NG & $\mathrm{NG}$ & $\mathrm{NG}$ & $\mathrm{NG}$ & $\mathrm{NG}$ & $(-25,25)$ \\
\hline [20] & $<-10$ & $<3$ & $56-66.2$ & $16.7 \%$ & $0.16 \lambda_{0}$ & 2 & $5-14.6$ & $>80 *$ & $\mathrm{NG}$ & NG & NG & NG & NG & NG & I \\
\hline [21] & $<-10$ & $<3$ & $52.2-72.2$ & $32.3 \%$ & $0.16 \lambda_{0}$ & 2 & $10.3-11.8$ & $\mathrm{NG}$ & NG & NG & NG & NG & NG & NG & l \\
\hline [22] & $<-10$ & $<3$ & $28.5-31.5$ & $10 \%$ & $0.13 \lambda_{0}$ & 4 & $12-13.5$ & $>67.5$ & NG & NG & NG & NG & NG & NG & l \\
\hline [23] & $<-10$ & $<3$ & $56.5-67$ & $17 \%$ & $0.16 \lambda_{0}$ & 2 & $11-13.5$ & $\leq 85$ & NG & NG & NG & NG & NG & NG & I \\
\hline $\begin{array}{l}\text { Presented } \\
1 \times 4 \text { Array }\end{array}$ & $<-10$ & $<3$ & $23.8-32.4$ & $30.6 \%$ & $0.16 \lambda_{0}$ & 4 & $11.2-12$ & $>70.3$ & $18-19$ & $24-30$ & $<3$ & $66-81$ & $55-75$ & $<4.3$ & $(-18,18)$ \\
\hline $4 \times 4$ Array & $<-10$ & $<3$ & $22.8-32$ & $33.6 \%$ & & & $17.1-18.5$ & $>60.9$ & $15-17$ & $28-30$ & $<3$ & $14-16$ & $19-21$ & $<3$ & / \\
\hline
\end{tabular}

(RBW: Relative bandwidth. $\eta_{T}$ : Total efficiency. NG: Not given in the reference. *: Simulated value. /: No such performance.)

Differentially-fed CP antenna in [21] has a similar wide operation bandwidth compared to our presented array antennas. However, because the sequential rotation feed networks are incorporated into the antenna aperture for high integration, the size of the radiation aperture is increased. This makes the antenna element difficult to be expanded into large array designs. Compared to the radiation performances of these reference antennas, the developed $1 \times 4$ linear array has a wide $3 \mathrm{~dB}$ AR beamwidth, which can ensure low AR while scanning to different angles. A wide $3 \mathrm{~dB}$ ARBW is achieved for the developed $4 \times 4$ planar array to cover the entire HPBW, and a stable CP antenna gain of 17.1-18.5 dBic is achieved. In addition, good radiation efficiencies of higher than $70.3 \%$ and $60.9 \%$ are achieved for the developed $1 \times 4$ linear array and $4 \times 4$ planar array, respectively.

\section{CONCLUSION}

This paper presents a wideband differentially-fed CP antenna for mmWave applications. The wideband $\mathrm{CP}$ radiation is realized by elaborately exciting a stepped corner-shaped slot, which is composed of two wide open slots at the ends and a narrow slot at the center. Combining the proper excitation using top microstrip feed line, the narrow slot can work as a phase delay line, and provide equal magnitude and quadrature phase to the two orthogonally arranged wide open slots for good $\mathrm{CP}$ radiation. Based on this design concept and the results of the antenna element, a $1 \times 4$ linear array and a $4 \times 4$ planar array were designed, fabricated, and measured for performance verification. Both the simulated and measured S-parameters and radiation patterns agree well with each other. The measured results show that wide overlapped impedance and AR bandwidths of 23.8-32.5 GHz and 22.8-32 GHz are achieved for the fabricated linear and planar arrays, respectively. In addition, the linear array has the scanning beams covering $-27^{\circ}$ to $27^{\circ}$, and the planar array has a high and stable gain of 17.1-18.5 dBic. Therefore, the presented CP antenna can be a good candidate for mmWave applications.

\section{APPENDIX}

To derive the transmission coefficient $S_{21}$ of the equivalent circuit shown in Fig. 2, this circuit can be simplified into a two-port network as shown in Fig. A1. So the ABCD parameters of the two-port network in the figure can be calculated as

$$
\left[\begin{array}{ll}
A & B \\
C & D
\end{array}\right]=\left[\begin{array}{cc}
1 & 0 \\
\frac{Z_{1}+j Z_{s} \tan \theta_{1}}{Z_{1}\left(Z_{s}+j Z_{1} \tan \theta_{1}\right)} & 1
\end{array}\right]
$$

Then, the transmission coefficient $S_{21}$ can be calculated by converting the ABCD matrix into an $S$ matrix, that is

$$
S_{21}=\frac{2 \sqrt{Z_{0} Z_{s}}}{Z_{s}+\frac{Z_{0} Z_{s}\left(Z_{1}+j Z_{s} \tan \theta_{1}\right)}{Z_{1}\left(Z_{S}+j Z_{1} \tan \theta_{1}\right)}+Z_{0}}
$$

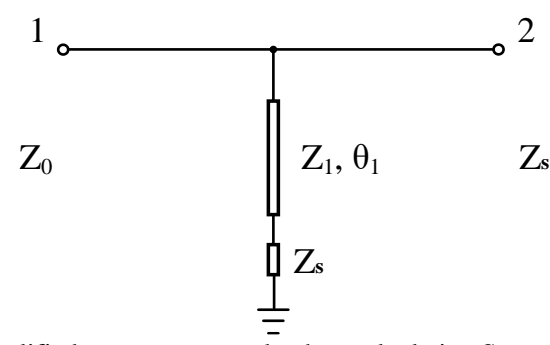

Fig. A1. Simplified two-port network when calculating $\mathrm{S}_{21}$.

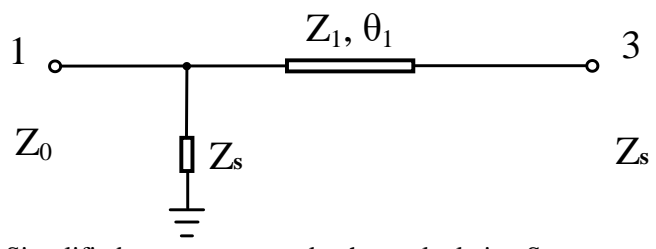

Fig. A2. Simplified two-port network when calculating $S_{31}$.

Similarly, to derive the transmission coefficient $S_{31}$, the equivalent circuit shown in Fig. 2 can be simplified into another two-port network as shown in Fig. A2. The ABCD parameters of the two-port network in the figure can be calculated as

$$
\begin{aligned}
& {\left[\begin{array}{ll}
A & B \\
C & D
\end{array}\right]=\left[\begin{array}{ll}
1 & 0 \\
\frac{1}{Z_{s}} & 1
\end{array}\right]\left[\begin{array}{cc}
\cos \theta_{1} & j Z_{1} \sin \theta_{1} \\
\frac{j \sin \theta_{1}}{Z_{1}} & \cos \theta_{1}
\end{array}\right]} \\
& =\left[\begin{array}{cc}
\cos \theta_{1} & j Z_{1} \sin \theta_{1} \\
\frac{\cos \theta_{1}+j \sin \theta_{1}}{Z_{1}} & \frac{j Z_{1} \sin \theta_{1}}{Z_{s}}+\cos \theta_{1}
\end{array}\right]
\end{aligned}
$$

Then, the transmission coefficient $S_{31}$ can be calculated by converting the ABCD matrix into an $\mathrm{S}$ matrix, that is 


$$
S_{31}=\frac{2 \sqrt{Z_{0} Z_{s}}}{\left(Z_{s}+Z_{0}\right) \cos \theta_{1}+\frac{j Z_{1}\left(Z_{s}+Z_{0}\right) \sin \theta_{1}}{Z_{s}}+\frac{Z_{0} Z_{s} e^{j \theta_{1}}}{Z_{1}}}
$$

\section{REFERENCES}

[1] W. Hong et al., "Multibeam antenna technologies for 5G wireless communications," IEEE Trans. Antennas Propag., vol. 65, no. 12, pp. 6231-6249, Dec. 2017.

[2] S. Gao, Q. Luo, and F. Zhu, Circularly polarized antennas. J Hoboken, NJ, USA: Wiley, 2013.

[3] G. Feng, L. Chen, X. Xue and X. Shi, "Broadband circularly polarized crossed-dipole antenna with a single asymmetrical cross-loop," IEEE Antennas Wireless Propag. Lett., vol. 16, pp. 3184-3187, 2017.

[4] Y. M. Pan, W. J. Yang, S. Y. Zheng and P. F. Hu, "Design of wideband circularly polarized antenna using coupled rotated vertical metallic plates," IEEE Trans. Antennas Propag., vol. 66, no. 1, pp. 42-49, Jan. 2018.

[5] R. Xu, J. Li and W. Kun, "A broadband circularly polarized crossed-dipole antenna," IEEE Trans. Antennas Propag., vol. 64, no. 10, pp. 4509-4513, Oct. 2016.

[6] L. Wen et al., "A wideband series-fed circularly polarized differential antenna by using crossed open slot-pairs," IEEE Trans. Antennas Propag., vol. 68, no. 4, pp. 2565-2574, April 2020.

[7] Z. Zhang, N. Liu, J. Zhao and G. Fu, "Wideband circularly polarized antenna with gain improvement," IEEE Antennas Wireless Propag. Lett., vol. 12, pp. 456-459, 2013.

[8] W. Hu et al., "Wideband circularly polarized antenna using single-arm coupled asymmetric dipoles," IEEE Trans. Antennas Propag., vol. 68, no. 7, pp. 5104-5113, July 2020.

[9] H. D. Li, X. Y. Du, J. Y. Yin, J. Ren and Y. Yin, "Differentially fed dual-circularly polarized antenna with slow wave delay lines," IEEE Trans. Antennas Propag., vol. 68, no. 5, pp. 4066-4071, May 2020.

[10] L. Wen, S. Gao, Q. Luo, W. Hu and Y. Yin, "Wideband dual circularly polarized antenna for intelligent transport systems," IEEE Trans. Veh. Technol., vol. 69, no. 5, pp. 5193-5202, May 2020.

[11] M. Li and K. Luk, "Low-cost wideband microstrip antenna array for 60-GHz applications," IEEE Trans. Antennas Propag., vol. 62, no. 6, pp. 3012-3018, June 2014.

[12] T. Zhang, Y. Zhang, L. Cao, W. Hong and K. Wu, "Single-layer wideband circularly polarized patch antennas for Q-Band applications," IEEE Trans. Antennas Propag., vol. 63, no. 1, pp. 409-414, Jan. 2015.

[13] X. Bai, S. Qu, S. Yang, J. Hu and Z. Nie, "Millimeter-wave circularly polarized tapered-elliptical cavity antenna with wide axial-ratio beamwidth," IEEE Trans. Antennas Propag., vol. 64, no. 2, pp. 811-814, Feb. 2016.

[14] Q. Wu, J. Hirokawa, J. Yin, C. Yu, H. Wang and W. Hong, "Millimeter-wave multibeam endfire dual-circularly polarized antenna array for 5G wireless applications," IEEE Trans. Antennas Propag., vol. 66, no. 9, pp. 4930-4935, Sept. 2018.

[15] Y. Yang, B. Sun and J. Guo, "A low-cost, single-layer, dual circularly polarized antenna for millimeter-wave applications," IEEE Antennas Wireless Propag. Lett., vol. 18, no. 4, pp. 651-655, April 2019.

[16] Y. Yang, B. Sun and J. Guo, "A single-layer wideband circularly polarized antenna for millimeter-wave applications," IEEE Trans. Antennas Propag., vol. 68, no. 6, pp. 4925-4929, June 2020.

[17] W. Zhang, Y. P. Zhang, M. Sun, C. Luxey, D. Titz and F. Ferrero, "A $60-\mathrm{GHz}$ circularly-polarized array antenna-in-package in LTCC technology," IEEE Trans. Antennas Propag., vol. 61, no. 12, pp. 6228-6232, Dec. 2013.

[18] A. Dadgarpour, M. Sharifi Sorkherizi and A. A. Kishk, "High-efficient circularly polarized magnetoelectric dipole antenna for $5 \mathrm{G}$ Applications using dual-polarized split-ring resonator lens," IEEE Trans. Antennas Propag., vol. 65, no. 8, pp. 4263-4267, Aug. 2017.

[19] Z. Gan, Z. Tu, Z. Xie, Q. Chu and Y. Yao, "Compact wideband circularly polarized microstrip antenna array for $45 \mathrm{GHz}$ application," IEEE Trans. Antennas Propag., vol. 66, no. 11, pp. 6388-6392, Nov. 2018

[20] D. J. Bisharat, S. Liao and Q. Xue, "High gain and low cost differentially fed circularly polarized planar aperture antenna for broadband millimeter-wave applications," IEEE Trans. Antennas Propag., vol. 64, no. 1, pp. 33-42, Jan. 2016.
[21] X. Ruan and C. H. Chan, "A circularly polarized differentially fed transmission-line-excited magnetoelectric dipole antenna array for $5 \mathrm{G}$ applications," IEEE Trans. Antennas Propag., vol. 67, no. 3, pp. 2002-2007, March 2019.

[22] M. M. M. Ali and A. Sebak, "Printed rgw circularly polarized differential feeding antenna array for $5 \mathrm{G}$ communications," IEEE Trans. Antennas Propag., vol. 67, no. 5, pp. 3151-3160, May 2019.

[23] D. J. Bisharat, S. Liao and Q. Xue, "Circularly polarized planar aperture antenna for millimeter-wave applications," IEEE Trans. Antennas Propag., vol. 63, no. 12, pp. 5316-5324, Dec. 2015.

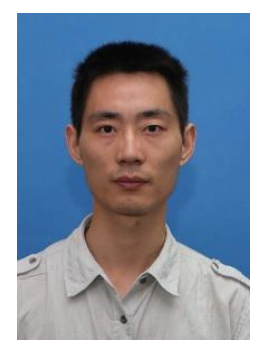

Lehu Wen received Ph.D. degree in electronic engineering from the University of Kent, Canterbury, U.K, in 2020.

$\mathrm{He}$ is currently a Research Associate with the School of Engineering and Digital Arts, University of Kent, Canterbury, U.K. His research interests include wideband dual-polarized antennas, circularly polarized antennas, tightly coupled array antennas, and mobile terminal antennas.

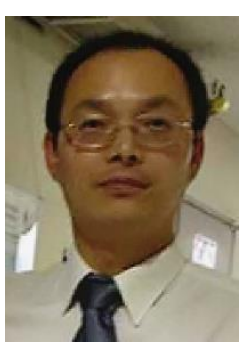

Steven Gao (Fellow, IEEE) received the Ph.D. degree in microwave engineering from Shanghai University, Shanghai, China, in 1999.

$\mathrm{He}$ is currently a Full Professor and Chair in RF and Microwave Engineering, and the Director of Graduate Studies at the School of Engineering and Digital Arts, University of Kent, UK. His current research interests include smart antennas, phased arrays, MIMO, satellite antennas, satellite communications, UWB radars, synthetic aperture radars, and mobile communications. $\mathrm{He}$ is currently an Associate Editor of the IEEE TRANSACTIONS ON ANTENNAS AND PROPAGATION.

Wei Hu (Member, IEEE) is currently an Associate Professor with the National Key Laboratory of Antennas and Microwave Technology, Xidian University, Xi'an, China.

Qi Luo (Senior Member, IEEE) is currently a Senior Lecturer with the School of Physics, Engineering and Computer Science, University of Hertfordshire, Hatfield, U.K.

Xue-Xia Yang (Senior Member, IEEE) is currently a Professor with the School of Communication and Information Engineering, Shanghai University, Shanghai, China.

Benito Sanz-Izquierdo is currently a Senior Lecturer with the School of Engineering and Digital Arts, University of Kent, Canterbury, U.K. 